\title{
MACA (Lepidium meyenii WALP), UNA REVISIÓN SOBRE SUS PROPIEDADES BIOLÓGICAS
}

\author{
Gustavo F. Gonzales $1,2, a$, Leonidas Villaorduña ${ }^{3, b}$, Manuel Gasco ${ }^{1,2, c}$, \\ Julio Rubio ${ }^{1, d}$, Carla Gonzales $1,2, \mathrm{e}$
}

RESUMEN

La maca (Lepidium meyenii) es una planta que crece sobre los 4000 metros de altitud en los Andes Centrales del Perú, presenta diferentes variedades de acuerdo al color de su hipocótilo. La presente revisión resume los resultados de estudios sobre los efectos de la maca en la función sexual, la espermatogénesis, la función reproductiva femenina, la memoria, la depresión y la ansiedad, como energizante y contra la hiperplasia benigna de próstata, osteoporosis y síndrome metabólico. Se discute también su efecto antienvejecimiento y la seguridad en su consumo. Se han demostrado diferencias en el efecto de las variedades negra, amarilla y roja de maca. La maca negra es la que mejores resultados presenta sobre la espermatogénesis, la memoria y contra la fatiga, mientras que la maca roja es la variedad que mejor revierte la hiperplasia benigna de próstata y la osteoporosis inducida experimentalmente. Además, la maca reduce los niveles de glucosa, y su consumo se relaciona con la reducción de la presión arterial y un mejor puntaje de salud. Estudios experimentales han demostrado que el consumo a corto como a largo plazo no muestra toxicidad tanto in vivo como in vitro. A pesar que los estudios experimentales han demostrado que la maca presenta diversos efectos benéficos, son necesarios más estudios clínicos para confirmar estos resultados.

Palabras clave: Espermatogénesis; Hiperplasia benigna prostática; Osteoporosis; Fatiga; Toxicidad (fuente: DeCS BIREME).

\section{MACA (Lepidium meyenii WALP), A REVIEW OF ITS BIOLOGICAL PROPERTIES}

\begin{abstract}
Maca (Lepidium meyenii) is a plant that grows above 4000 altitude meters in Peru's Central Andes; it has different varieties according to the color of the hypocotyl. This review summarizes the results of studies about the effects of maca on sexual function, spermatogenesis, female reproductive function, memory, depression and anxiety, and energy as well as effects on benign prostatic hyperplasia, osteoporosis and metabolic syndrome. Its anti-aging effect is also discussed as well as safety in consumption. Differences have been shown between the effects of the black, yellow and red maca varieties. Black maca shows the best results on spermatogenesis, memory and fatigue, while red maca is the variety that reverses the benign prostatic hyperplasia and experimentally induced osteoporosis. In addition, maca reduces the glucose levels, and its consumption is related to the lowering of blood pressure and an improved health score. Experimental studies have proven that short and long term consumption don't show in vivo and in vitro toxicity. Although experimental studies have shown that maca has diverse beneficial effects, more clinical studies are needed to confirm these results.
\end{abstract}

Key words: Spermatogenesis; Prostatic hyperplasia; Osteoporosis; Fatigue; Toxicity (source: MeSH NLM).

Facultad de Ciencias y Filosofía, Universidad Peruana Cayetano Heredia. Lima, Perú.

Instituto de Investigaciones de la Altura, Universidad Peruana Cayetano Heredia. Lima, Perú

Universidad Nacional Daniel Alcides Carrión. Cerro de Pasco, Perú.

Doctor en Medicina y doctor en Ciencias; ${ }^{b}$ doctor en Economía; ${ }^{c}$ magíster en Ciencias; ${ }^{d}$ licenciado en Biología; ${ }^{e}$ doctora en Ciencias Recibido: 11-09-13 Aprobado: 29-01-14

Citar como: Gonzales GF, Villaorduña L, Gasco M, Rubio J, Gonzales C. Maca (Lepidium meyenii Walp), una revisión sobre sus propiedades biológicas. Rev Peru Med Exp Salud Publica. 2014;31(1):100-10. 


\section{INTRODUCCIÓN}

La maca (Lepidium meyenii) pertenece a la familia Brassicaceae y crece en los Andes centrales del Perú por encima de los 4000 metros de altitud donde se cultiva desde hace más de 2000 años ${ }^{(1)}$. Sus primeras descripciones aparecen en las crónicas de la conquista del Perú (2-3). Se han descrito hasta trece variedades que van del blanco al negro ${ }^{(4)}$, se ha estudiado variedades, como la negra y la roja las cuales mostraron distintas propiedades ${ }^{(5)}$. Estas diferencias de las propiedades biológicas parecen depender de las diferencias del suelo de cultivo, antes que de los morfotipos ${ }^{(6)}$; sin embargo, contrasta el hecho que todas las variedades se presenten en un mismo suelo ${ }^{(4)}$.

La adaptación de esta planta a lugares tan adversos determina que su característica morfológica incluya una parte aérea muy pequeña y una zona reservante (hipocótilo) voluminosa que se encuentra en el interior de la tierra de cultivo. Por ello, no es de sorprender que una zona de cultivo de maca se parezca a un campo de fútbol ${ }^{(7)}$. El interés por esta planta ha traspasado nuestras fronteras de tal manera que se ha intentado cultivarla fuera de su hábitat natural en Europa, sin resultados halagadores ${ }^{(8)}$.

Su ciclo de vida tiene dos etapas bien definidas, la primera denominada la vegetativa donde se produce la raíz reservante o hipocótilo, que es utilizada como alimento por la población. La otra etapa conocida como la reproductiva consiste en la producción de semilla botánica; ambas etapas se completan en dos años consecutivos y, por ello, se conoce como un cultivo bienal.

El manejo agronómico de la etapa vegetativa de la maca consiste en preparar el terreno, analizar las características del suelo, hacer la siembra, y el mantenimiento del cultivo con las labores culturales, la cosecha y el secado al natural de los hipocótilos. Tradicionalmente, se consumen los hipocótilos de maca luego de una cocción, y la fracción activa es la fase acuosa ${ }^{(7)}$.

La etapa reproductiva de la maca consiste en seleccionar los mejores hipocótilos en estado fresco, prepararlos para su estado de reposo o dormancia; se les corta las hojas y se las desinfecta, luego se colocan en javas y se dejan en un ambiente seco, fresco y no expuestas a radiación solar. En el estado de reposo se debe tener cuidado del ataque de enfermedades y dotarlo con agua limpia para conservar la humedad necesaria. En este estado, los hipocótilos deben permanecer de dos meses a dos meses y medio.

Cuando los brotes están de dos a tres centímetros de tamaño se pueden trasplantar en el campo adecuadamente preparado, con distanciamientos de 80 centímetros entre plantas y lo mismo entre surcos. Al llegar los cinco meses de trasplantados los hipocótilos, y cuando las semillas ya están maduras, se inicia la cosecha de la maca colocando las inflorescencia de la planta denominada rosetas, luego el secado de las rosetas $y$, finalmente, se procede a la separación de la semilla de las ramas, hojas e impurezas.

En la fracción acuosa de la maca se han encontrado azucares libres, aminoácidos con alto componente de prolina, uridina, ácido málico y una fracción conteniendo glucosinolatos ${ }^{(9,10)}$, mientras que en la fase no polar hay macaenos y macamidas (ácidos grasos poliinsaturados y sus correspondientes amidas) ${ }^{(9)}$. En maca seca el contenido de macaenos oscila entre 0,09 y $0,45 \%{ }^{(11)}$. Las principales macamidas identificadas son: $n$-bencil pentadecanamida, $n$-bencil hexadecanamida, n-(3metoxibencil)-hexadecanamida, n-bencil-hepta decanamida, n-bencil-(9z)-octadecanamida, n-bencil-9-oxo-(12z)-octodecamida, n-bencil-(9z,12z)octadecadienamida, n-bencil-9-oxo-(12z,15z)-octadecadienamida, n-bencil-13-oxo-(9E,11E)-octadecadienamida, n-bencil-5-oxo-(6E,8E)-octadecadienamida, n-3-metoxibencil-9z-octadecanamida, n-bencil-(15z)-tetracosenamida, metoxi-n-bencil-(9z,12z)-octadecadienamida, n-3-metoxibencil-(9z-12z)-octadecadienamida, n-bencil-(9z,12z,15z)octadecatrienamida, metoxi-n-bencil-(9z,12z,15z)-octadecatrienamida,n-3-metoxibencil-(9z,12z,15z)-octadecatrienamida y la n-benciloctadecanamida ${ }^{(12,13)}$.

En la cosecha, los hipocótilos de maca contienen seis glucosinolatos: 5-metilsulfinilpentil, 4-hidroxibencil, bencil, 3-metoxibencil, 4-hidroxi-3-indolilmetil y 4-metoxi3 -indolilmetil glucosinolato, de los cuales el bencil glucosinolato representa el $80 \%$ del total. Los hipocótilos de maca negra tienen en la cosecha más cantidad de 5 -metilsulfinilpentil glucosinolato que la maca roja ${ }^{(14)}$.

Tradicionalmente se consume la maca como mezcla de diferentes colores ${ }^{(15)}$. Actualmente, la maca ya no es solamente consumida en las zonas de los Andes centrales, donde se produce, sino que hay un uso diseminado en todo el país ${ }^{(16)}$. El interés comercial sobre este producto, tanto en el Perú como en diferentes partes de mundo, hace necesario conocer sobre sus propiedades biológicas. La presente revisión muestra algunas características sobre sus propiedades biológicas y toxicidad basados en investigaciones científicas

\section{METODOLOGÍA}

Se realizó una revisión narrativa no sistemática; la revisión se concentró primordialmente en artículos 
publicados en revistas científicas que evalúen las propiedades biológicas de la maca desde el año 2000, la estrategia de búsqueda incluyó términos como "Lepidium meyenii, Lepidium peruvianum" y "maca" en PubMed, Google scholar y ScienceDirect.

\section{MACA Y FUNCIÓN SEXUAL}

La testosterona incrementa el deseo sexual en ambos sexos y favorece la erección en varones. La disminución en los niveles de testosterona sérica se asocia a menor deseo sexual y a disfunción eréctil. El tratamiento con testosterona sería eficaz en estos casos, pero el temor a sus efectos adversos hace que su uso no sea común, es por ello que se busca otras opciones de tratamiento ${ }^{(17)}$.

La maca, particularmente extractos lipídicos, mejora la conducta sexual en roedores (18-20); sin embargo estos resultados no han podido ser reproducidos en posteriores estudios ${ }^{(21,22)}$. La maca gelatinizada $(1,5 \mathrm{o}$ 3,0 g/día) en varones normales, mejora el deseo sexual a partir de las 8 semanas de administración, tiempo largo para un tratamiento sobre mejora del deseo sexual (23). En cambio, el uso de extracto de maca mejora el deseo sexual en varones deportistas a las dos semanas de tratamiento ${ }^{(24)}$. En un estudio doble ciego usando un extracto de maca seca (2,4 g/día) por doce semanas se observa un pequeño pero significante efecto de la maca sobre la disfunción eréctil leve ${ }^{(25)}$.

Dos ensayos clínicos sugieren un efecto favorable de la maca sobre la disfunción sexual o en el deseo sexual en mujeres menopáusicas sanas ${ }^{(27)}$ o adultos varones sanos ${ }^{(23)}$, respectivamente, mientras que en otro ensayo clínico los revisores manifiestan que no hay efecto de la maca en ciclistas sanos. Sin embargo, los resultados del estudio, muestran que el extracto de maca mejora significativamente el deseo sexual comparado con los valores basales $(p=0,01)$, o con el placebo $(p=0,03)^{(24)}$. Una revisión sistemática que evalúa el efecto de la maca en la mejora de la función sexual en humanos manifiesta que la evidencia aún es limitada ${ }^{(26)}$.

En un estudio clínico sin comparación con placebo, pacientes con disfunción sexual inducida por el uso de inhibidores de la recaptura de serotonina (SSRI), que consumían 3,0 g/día de maca tuvieron una mejoría en las pruebas de función sexual, lo que no ocurrió con la dosis de 1,5 g/día de maca; siendo el consumo de maca bien tolerada ${ }^{(28)}$. La evaluación del efecto del consumo de maca sobre la función sexual todavía requiere de mayores estudios con diseños más adecuados y rigurosos.

\section{MACA Y ESPERMATOGÉNESIS}

El extracto acuoso obtenido luego de la cocción de los hipocótilos de maca y el extracto hidroalcohólico, son capaces de aumentar los estadios de mitosis de la espermatogénesis en rata, luego de 14 días de tratamiento ${ }^{(29,30)}$. Igualmente, hay un efecto dosisrespuesta que mejora los estadlos de espermiación (VII-VIII) y mitosis en rata, luego de 7 días de tratamiento ${ }^{(31)}$. Después de 42 días, la maca negra mejoró la producción diaria de espermatozoides y aumentó la movilidad en espermatozoides $(p<0,05)$. No se observó efecto de la maca roja mientras que la maca amarilla tuvo un efecto intermedio ${ }^{(32)}$.

La administración por 84 días de maca negra o amarilla aumenta el conteo de espermatozoides en el epidídimo; las tres variedades (negra, amarilla y roja) aumentan el conteo de espermatozoides en el conducto deferente, sin afectar el número de espermatozoides en los testículos. Estos resultados sugerirían una acción de la maca modulando el conteo de espermatozoides ${ }^{(33)}$.

Los mayores efectos sobre la espermatogénesis se ha observado con la fracción etil acetato, extraída de los hipocótilos de la maca negra; sin embargo, este efecto no fue superior al extracto hidroalcóholico total ${ }^{(34)}$.

En toros, la suplementación de la alimentación con maca parece mejorar la cantidad, calidad e integridad del DNA de los espermatozoides ${ }^{(22)}$. Igualmente, la mezcla de extractos de maca negra con hojas de yacón revierten la reducción de espermatozoides y el incremento de la glucosa inducida por la estreptozotocina ${ }^{(35)}$.

La administración de maca gelatinizada a nueve varones sanos por 4 meses, muestra en dosis de $1,5 \mathrm{~g}$ o 3,0 g un aumento del volumen seminal, del conteo y de la movilidad de espermatozoides ${ }^{(36)}$. Luego de 4 meses de tratamiento, el número de espermatozoides móviles se incrementa de 87,72 \pm 19,87 millones (media \pm error estándar de la media) a $183,16 \pm 47,84$ millones $(p<0,05)^{(36)}$.

Los niveles de hormona luteinizante (LH), folículo estimulante (FSH), prolactina, estradiol y testosterona no fueron afectados por el tratamiento con maca ${ }^{(37)}$.

La falta de respuesta de la testosterona sérica o la falta de activación del receptor de andrógenos por acción de la maca ${ }^{(27,38)}$ sugiere que los efectos de la maca sobre la calidad o cantidad de los espermatozoides se producen por otra vía diferente a testosterona y su receptor o a FSH. Estudios in vitro indican que tanto los extractos metanólicos como acuosos de maca muestran actividad estrogénica en líneas celulares MCF-7 ${ }^{(9)}$, y al 
no haber encontrado acción en los receptores alfa estrogénicos ${ }^{(27)}$ se sugiere una acción en los receptores beta de estrógenos, lo cual necesita aún ser demostrado.

\section{EFECTO DE LA MACA SOBRE LA FUNCIÓN REPRODUCTIVA FEMENINA}

La maca no afecta la tasa de implantación en ratonas ${ }^{(39,40)}$, ni genera mayor número de óvulos por ciclo ${ }^{(41)}$, ni afecta el desarrollo normal de los embriones preimplantacionales ${ }^{(42)}$. Sin embargo, las ratonas que recibieron extracto acuoso de maca presentaron un mayor número de crías que las del grupo control. Lo mismo se ha observado en cobayos tratados con $90 \mathrm{~g}$ de maca/día durante 100 días ${ }^{(43)}$. En peces, también se ha mostrado que la maca favorece la supervivencia de alevinos y juveniles ${ }^{(44,45)}$.

Se ha reportado que el uso de fitoestrógenos en la dieta, acelera el tiempo de apertura vaginal en las ratonas ${ }^{(46)}$. El tratamiento con maca no afecta esta variable, por lo que el consumo de hipocótilos de maca no afecta la maduración sexual ${ }^{(40)}$.

Estudios en poblaciones que tradicionalmente consumen maca indican que esta planta es consumida incluso durante la gestación ${ }^{(15)}$. Sin embargo, se hace necesario realizar estudios para determinar su seguridad, aun cuando se consume en etapas tempranas de la gestación.

\section{MACA Y HORMONAS SEXUALES}

Se han realizado estudios en ratas, ratones y humanos tratando de evaluar el efecto de la administración de la maca en diferentes formas sobre los niveles de las hormonas sexuales.

En ratonas, la maca no afecta los niveles séricos de estradiol; sin embargo, los niveles de progesterona se encuentran aumentados ${ }^{(39)}$. Se ha sugerido que los efectos observados en ratas preñadas, donde la maca protege de la mortalidad embrionaria, podrían deberse a la progesterona ${ }^{\left({ }^{40}\right)}$. Estudios en ratas también muestran un efecto de la maca en aumentar los niveles de progesterona. En ratas ovariectomizadas más bien se observa un efecto inhibitorio de la maca sobre los niveles de progesterona ${ }^{(47)}$. Los niveles de estradiol se reducen por efecto de la maca en ratas normales y en ovariectomizadas ${ }^{(47)}$.

En animales machos también hay datos discordantes, aunque la mayoría de estudios apuntan a concluir que la maca no afecta los niveles de testosterona y estradiol sérico en ratas y ratones.
En un estudio en ratones administrados con maca por 30 días, se concluye que la maca con valores de testosterona de $4,37 \pm 0,69 \mathrm{ng} / \mathrm{mL}$ con respecto al control de $3,39 \pm 0,53 \mathrm{ng} / \mathrm{mL}$ muestra diferencia significativa en esta hormona (39); sin embargo, al calcular mediante la prueba $t$ de Student se puede demostrar que no hay diferencia significativa. Ratas machos tratadas con 480 $96 \mathrm{mg} /$ día con un extracto hidroalcohólico de maca por 21 días no afectan los niveles de testosterona sérica (30). En otro estudio donde se utiliza extracto acuoso hervido de maca en dosis de 0,$01 ; 0,1 ; 1$ y 5 gramos maca $/ \mathrm{kg}$ peso corporal de ratas adultas, muestra que los niveles de testosterona sérica y la relación testosterona/estradiol se reducen con $0,1 \mathrm{~g}$ extracto de maca por 7 días, pero no con las otras dosis; los niveles de estradiol en suero fueron similares con respecto al grupo control (31).

La administración de extractos acuosos hervidos de maca roja, negra o amarilla por 7 días no modificaron los niveles de testosterona sérica o estradiol con respecto al control; sin embargo, el grupo de maca amarilla presentó mayores niveles de testosterona sérica que el tratado con maca negra debido a dos ratas que tenían niveles altos de testosterona ${ }^{(5)}$. En un posterior estudio se observó que los niveles de testosterona intratesticular y los niveles séricos de testosterona fueron similares en el grupo que recibió maca negra por 7 días que en el grupo control ${ }^{(48)}$.

Se han evaluado también los niveles de testosterona en otras situaciones, una evaluó el efecto de la administración del acetato de plomo ${ }^{(49)}$ y otro para evaluar el efecto de la altura ${ }^{(50)}$. La maca no revirtió el efecto deletéreo del plomo sobre los niveles de testosterona sérica ${ }^{(49)}$. En la altura se observa un mayor valor de testosterona en el grupo tratado con maca con respecto al control sin maca; a los 14 y 21 días de exposición a la altura los valores de testosterona sérica son similares entre el control y el grupo tratado con maca ${ }^{(50)}$. Se ha observado en estudios experimentales que la testosterona aumenta durante la exposición a la altura ${ }^{(51)}$ por lo que los resultados pueden indicar que la maca favorecería la aclimatación a la altura.

Los niveles de testosterona o estradiol no se modifican en varones aparentemente sanos luego de cuatro meses de tratamiento con 1,5 3 gramos diarios de maca ${ }^{(36)}$. En un estudio en humanos que recibieron maca durante tres meses $(1,5 \circ 3 \mathrm{gr} / \mathrm{dí})$ ), los niveles de 17 hidroxiprogesterona fueron más altos en el grupo con maca ( $3 \mathrm{gr} / \mathrm{día}$ ) a las dos semanas de tratamiento; sin embargo, en el análisis de varianza de dos vías se muestra que el efecto del tiempo del tratamiento fue no significativo ${ }^{(37)}$. Lo que sugiere que la diferencia observada era debido a los valores basales más que a un efecto real de la maca. En este mismo grupo, los 
niveles de testosterona o estradiol en suero, no se modificó a ningún tiempo de tratamiento $(0,2,4,8$ o 12 semanas) por efecto de la maca (1,5 o $3 \mathrm{gr} /$ día) ${ }^{(37)}$.

En mujeres perimenopáusicas y en posmenopáusicas se observó una elevación en los niveles de estradiol $(52,53)$, que contrasta con lo presentado por el mismo autor en ratas ${ }^{(47)}$.

\section{EFECTODE LAMACA SOBREMEMORIA, DEPRESIÓN Y ANSIEDAD}

Un estudio de los efectos de la maca sobre la función cognitiva evaluó la acción de tres variedades de maca (roja, negra y amarilla) sobre el aprendizaje en ratones ovariectomizados utilizando la prueba de "búsqueda de agua". Dicho estudio determinó que la maca negra tiene el mejor efecto sobre el aprendizaje espacial ${ }^{(54)}$.

Un segundo estudio determinó que los tratamientos con los extractos acuoso poshervido e hidroalcohólico de maca negra, revertían el daño cognitivo inducido por la escopolamina en ratones macho. La escopolamina es un antagonista de receptores muscarínicos que imita de manera aguda la enfermedad de Alzheimer ${ }^{(55)}$. Además, se demostró que ambos extractos de maca negra inhibieron la actividad de la acetilcolinesterasa, sin modificar los niveles de monoamino oxidasa ${ }^{(56)}$.

Estos resultados fueron confirmados utilizando ratones hembras donde los extractos acuoso poshervido e hidroalcohólico de maca negra fueron capaces de revertir el daño sobre la memoria y aprendizaje causado por la ovariectomía. Además, los extractos de maca negra disminuyeron los niveles de malonaldehído y acetilcolinesterasa sin modificar los niveles de monoamino oxidasa ${ }^{(57)}$.

Igualmente, el extracto hidroalcohólico de maca negra revirtió los efectos del alcohol (20\%) en ratones, de manera dosis-respuesta durante los ensayos de adquisición del escape en la prueba de nado de Morris. Más aun, el ácido ascórbico y la maca negra amenguaron los efectos deletéreos del etanol en las pruebas posentrenamiento ${ }^{(58)}$.

Un estudio en ratas recién destetadas y tratadas con extracto acuoso de maca amarilla por 15 días, demuestra que al aumentar la dosis disminuye la latencia de escape en la prueba de Morris. Además, la maca amarilla inhibió la butirilcolinesterasa y redujo la lipoperoxidación (daño oxidativo) en el cerebro ${ }^{(59)}$.

La actividad neuroprotectiva de la maca también ha sido estudiada usando modelos experimentales in vitro e in vivo. In vitro, el pretratamiento con el extracto pentánico de maca mejora la viabilidad de las neuronas de langostas, ante la exposición a peróxido de hidrógeno $\left(\mathrm{H}_{2} \mathrm{O}_{2}\right)$ de forma dependiente de la concentración.

En ratas se observó que el extracto pentánico presenta una potencial aplicación como neuroprotector en ratas con infarto cerebral por oclusión de la arteria cerebral media ${ }^{(60)}$. En humanos, la maca reduce los puntajes de depresión y ansiedad en varones adultos aparentemente sanos ${ }^{(7)}$. Igualmente, la maca $(3,5 \mathrm{~g} / \mathrm{d})$ por seis semanas reduce en mujeres posmenopáusicas los síntomas psicológicos, incluyendo ansiedad y depresión (27).

\section{MACA COMO ENERGIZANTE}

En ratas machos recién destetadas y tratadas con extracto acuoso de maca amarilla $(400,800$ o 1,200 mg maca/kg peso) durante 30 días, no se muestra efecto sobre la prueba de nado forzado que mide la resistencia física. La cocción se realizó por 30 minutos ${ }^{(61)}$.

Igualmente, hay efecto sobre la resistencia física suplementando con harina de maca fermentada ${ }^{(62)} \mathrm{O}$ con extracto acuoso de maca ${ }^{(63)}$ utilizando dosis muy altas. El extracto lipídico soluble de maca amarilla $(100 \mathrm{mg} / \mathrm{kg}$ ) durante tres semanas, aumentó la resistencia física, medida en términos del tiempo en que llega a la fatiga, en un $41 \%$ del valor del control ${ }^{(64)}$, en cambio, con el extracto hidroalcohólico de maca negra el incremento de la resistencia física es mayor al 500\%.

La administración de extracto de maca por 14 días de manera significativa y más rápida, mejora el tiempo de rendimiento en una carrera de ciclismo de $40 \mathrm{~km}$ comparado al valor basal $(p=0,01)$, pero no comparado al placebo $(p>0,05)^{(24)}$.

En diez futbolistas profesionales, que durante 60 días recibieron tres cápsulas de concentrado de maca fresca, con 500 mg cada una por día, se encuentra un incremento en promedio de $10,3 \%$ en el consumo de oxígeno luego de la administración ${ }^{(65)}$. Si bien ambos estudios en humanos muestran un efecto favorable del uso de la maca para la resistencia física, los incrementos son modestos.

\section{MACA E HIPERPLASIA BENIGNA DE PRÓSTATA}

La próstata, una glándula sexual accesoria, contribuye con el $30 \%$ del líquido seminal total $60 \%$ son proveídos por las vesículas seminales y $10 \%$ por las secreciones del epidídimo y de las glándulas bulbo-uretrales). Las 
secreciones de la próstata tienen como función proveer la capacidad fertilizante al espermatozoide. Su crecimiento y mantenimiento está regulado principalmente por los andrógenos, específicamente por la conversión de testosterona a dihidrotestosterona (DHT), reacción mediada por la enzima 5-alfa-reductasa ${ }^{(66)}$.

A partir de los 40 años, en casi la mitad de los varones, se observa un crecimiento de la próstata conocido como hiperplasia benigna de próstata (HBP) que se caracteriza por signos y síntomas del tracto urinario bajo que afecta la calidad de vida ${ }^{(66)}$. Aunque los bloqueadores de receptores adrenérgicos alfa 1 , inhibidores de la 5 alfa reductasa como el finasteride, y la combinación de terapias que incluyen tamsulosina-dutasteride, doxazosina-finasteride, terazosina-finasteride, tolterodina-tamsulosina y rofecoxib-finasteride forman parte de la estrategia terapéutica de elección para la HBP, en los últimos años está apareciendo información sobre el uso de fitoterapia para esta enfermedad ${ }^{(67)}$. Entre ellas, se describe a la maca roja ${ }^{(67)}$.

En un estudio se evaluó el efecto de un extracto acuoso liofilizado de la maca roja en HPB inducida por Enantato de testosterona en ratas adultas de la cepa Holtzman y la maca roja fue capaz de revertir este efecto sobre la próstata, siguiendo un patrón dosis-respuesta, sin afectar el peso de las vesículas seminales. En este mismo estudio, un grupo control positivo recibió finasteride reduciendo tanto el peso de la próstata como de las vesículas seminales. Estos resultados sugieren que la maca no actúa sobre la 5 alfa reductasa, sino probablemente dentro de la célula en factores de transcripción o tal vez en la vía estrogénica ${ }^{(68)}$.

La maca roja reduce los niveles de zinc intraprostático (incrementados en HPB) cuando es administrada del día 1 al día 14 teniendo en cuenta que el enantato de testosterona es aplicado el día 1 y el día $7{ }^{{ }^{69}}$. En la actualidad es importante realizar un ensayo clínico en pacientes que sufren de HBP para poder determinar su eficacia en seres humanos.

\section{MACA Y OSTEOPOROSIS}

Durante la posmenopausia hay una caída de estrógenos, la cual desencadena una serie de eventos que conducen a la osteoporosis ${ }^{(70)}$. Si bien la terapia hormonal de reemplazo (THR) ha demostrado ser efectiva en la prevención de la pérdida de masa ósea y la reducción del índice de fracturas, el riesgo de tener efectos colaterales serios ha generado una disminución en su uso y la preferencia por los bifosfonatos como el ácido alendrónico o el ácido zoledrónico ${ }^{(70)}$. La fitoterapia se ha sugerido como alternativa para la THR ${ }^{(71)}$.
La maca roja, al tener un efecto protector sobre órganos regulados por un balance de hormonas esteroideas, donde la actividad estrogénica cumple un rol importante, sugiere que esta variedad podría tener un efecto significativo sobre el balance homeostático de otras funciones reguladas por hormonas esteroideas, como es el metabolismo óseo.

En ratas ovariectomizadas tratadas con extractos etanólicos de maca a dosis orales de 0,096 y 0,24 g de extracto/kg peso corporal durante 28 semanas, se encontró que altas concentraciones del extracto etanólico de maca prevenían la perdida ósea ${ }^{(72)}$. La maca roja como la maca negra, pero no la amarilla, presentan efecto sobre la osteoporosis similar al estradiol ${ }^{(73)}$.

A diferencia del estradiol, ninguno de los tratamientos con las tres variedades de maca incrementó el peso uterino, lo que sugiere que la maca tiene una acción específica sobre dicho órgano ${ }^{(73)}$. El estradiol actúa a través de dos receptores alfa y beta. El receptor alfa de estradiol puede oponerse a la acción del receptor beta. Se sabe que el receptor beta de estradiol, si bien está ampliamente distribuido en el organismo, no está mayormente expresado en el útero ${ }^{(74)}$, por lo que se sugiere que la maca podría activar receptores beta estrogénicos.

Un estudio en los Andes centrales muestra que los consumidores de maca tienen menos tasas de fractura que aquellos de la misma zona que no consumen maca ${ }^{(15)}$, corroborando los resultados experimentales encontrados en animales ${ }^{(73)}$.

\section{MACA Y METABOLISMO}

El extracto hidroalcohólico de maca negra puede reducir en un $50 \%$ el valor de glucosa en sangre en ratas machos, en las que se ha inducido la diabetes por la administración de estreptozotocina ${ }^{(35)}$. La maca amarilla disminuye la glicemia y aumenta los niveles de insulina en ratas diabéticas inducidas con streptozotocina ${ }^{(75)}$. La maca administrada al $1 \%$ en la dieta de animales hipertrigliceridémicos mejora el perfil lipídico y aumenta la tolerancia a la glucosa ${ }^{(76)}$.

Un estudio aleatorizado controlado por placebo, y de 90 días de duración, evaluó los efectos de la maca en pacientes con síndrome metabólico, sin efectos favorables ${ }^{(77)}$.

En un estudio clínico en varones normales tratados con maca gelatinizada hubo una reducción de la presión arterial diastólica luego de 12 semanas de tratamiento ${ }^{(5)}$. Los pobladores que tradicionalmente consumen maca tienen menor presión arterial sistólica que aquellos que no la consumen (15). 


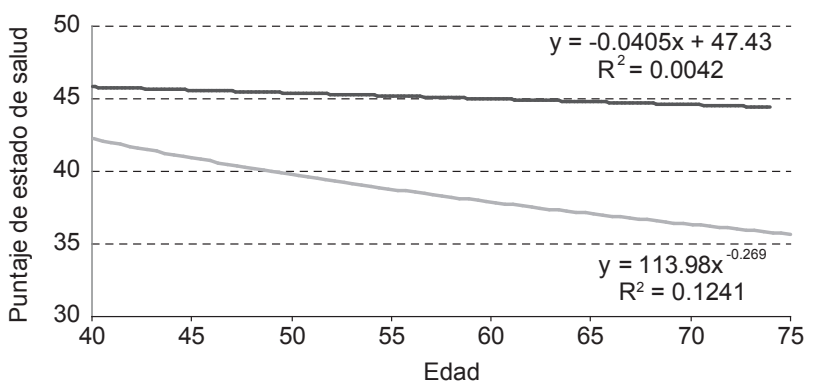

—Lineal (Consumen maca) _ Potencial (No consumen maca)

Figura 1. Puntajes de estado de salud en pobladores de Carhuamayo a $4100 \mathrm{~m}$ de altitud que consumen y que no consumen maca

Igualmente, la maca inhibe significativamente la enzima convertidora de angiotensina I (ACE) in vitro ${ }^{(78)}$. La maca contiene niveles altos de potasio ${ }^{(16)}$, nutriente importante para reducir el riesgo de hipertensión.

Se requieren de más estudios tanto experimentales como clínicos que demuestren el rol de la maca en el metabolismo de la glucosa, de los lípidos y de la regulación de la presión arterial.

\section{MACA EL ALIMENTO ANTIENVEJECIMIENTO}

En un estudio realizado en pobladores residentes a $4100 \mathrm{~m}$ de altitud, se encontró que en consumidores tradicionales de maca el puntaje de estado de salud basado en un cuestionario validado se mantiene desde los 40 a 75 años, a diferencia de la población que no consume maca, quienes presentan una disminución de dicho puntaje a medida que aumenta la edad (Figura 1) ${ }^{(15)}$.

La interleukina 6, un compuesto que cuando está aumentado en personas mayores se asocia al envejecimiento, se encuentra en menor cantidad en los sujetos de Junín residentes a $4100 \mathrm{~m}$ de altitud que consumen maca, esto se asocia a mejor puntaje de estado de salud y a un mayor éxito en la prueba de levantarse y sentarse cinco veces de una silla sin utilizar los brazos como soporte $(p<0,05)^{(79)}$.

\section{TOXICIDAD}

Los extractos acuosos y metanólicos de la maca no muestran hepatotoxicidad in vitro ${ }^{(9)}$. Igualmente, el extracto acuoso de maca en dosis de $1 \mathrm{~g}$ de maca liofilizada/kg en ratones, no altera el desarrollo normal de embriones preimplantados ${ }^{(42)}$. En ratas, las diferentes variedades de maca no muestran toxicidad aguda a dosis $\leq 17 \mathrm{~g}$ de hipocótilos de maca seca/kg. Igualmente, las ratas tratadas por 84 días con maca no muestran efectos adversos a dosis de $1 \mathrm{~g} / \mathrm{kg}$ de peso corporal, y la histología hepática fue similar al grupo control ${ }^{(33)}$.

En ratones la LD50 es mayor $15 \mathrm{~g}$ maca/ $\mathrm{kg}{ }^{(47)} \mathrm{sin}$ alteraciones histopatológicas en hígado, páncreas, bazo, testículos $u$ ovarios (53). Sin embargo, en pacientes con síndrome metabólico, la administración de maca en dosis de 0,6 g/día por 90 días incrementa moderadamente la enzima aspartato aminotransferasa (AST) y la presión arterial diastólica (77). Esto no ha podido ser confirmado en otros estudios que, más bien, muestran que la maca reduce la presión arterial tanto sistólica como diastólica ${ }^{(5,7,15,78)}$. Esto puede deberse al alto contenido en potasio (16) $\mathrm{o}$ a la actividad inhibidora de la enzima conversora de angiotensina descrita in vitro ${ }^{(78)}$. Igualmente, en estudios experimentales en animales hipercolesterolémicos la maca no solo no es tóxico, sino que protege mejor contra el daño hepático de la hipercolesterolemia que la atorvastatina ${ }^{(80)}$.

La demostración de la presencia de alcaloides como la (1R,3S)-1-metil tetrahidro- $\beta$-carbolina-3-ácido carboxílico en la maca ha motivado preocupación en su consumo, particularmente en el mercado europeo, por la sugerencia de un efecto tóxico ${ }^{(10)}$, por lo que es necesario mayores estudios para evaluar la fracción butanólica que contiene a este alcaloide y así comprobar su eficacia y seguridad.

\section{OTRAS PROPIEDADES}

Los estudios científicos muestran una variedad de funciones asociadas al consumo de la maca, ya sea en su preparación tradicional, en su forma gelatinizada o como extractos. La maca, según refieren los estudios, podría favorecer la respuesta del organismo ante situaciones de estrés ${ }^{(61,81)}$. Así, por ejemplo, el extracto metanólico de maca es capaz de atenuar, o de eliminar, variaciones de la homeostasis producida por estrés ${ }^{\left({ }^{11}\right)}$ lo que insinúa otorgar a la maca un rol adaptógeno. Se ha sugerido, además, que las actividades biológicas de la maca se deban a su actividad antioxidante ${ }^{(45,82,83)}$ y contenido de polifenoles. En la maca, la glucotropaelina representa el $79 \%$ del total de glucosinolatos y catequinas, y las galocatequinas el $97 \%$ del total de fenoles ${ }^{(84)}$. Sin embargo, comparativamente con diez plantas peruanas, si bien se encuentra asociación entre contenido de polifenoles y la actividad antioxidante, es de notar que la maca presenta los valores más bajos de polifenoles, carotenos y actividad antioxidante ${ }^{(85)}$. El hallazgo de que el camu camu (Myriciria dubia) tiene mayor contenido de polifenoles y mayor actividad antioxidante 
que la maca negra, a pesar que ambos actúan mejorando el conteo de espermatozoides, sugieren que el efecto de la maca no es a través de su actividad antioxidante. Queda una tarea por investigar para conocer qué compuestos participan en la actividad biológica de la maca y qué mecanismos emplea en el organismo para cumplir con dichas funciones ${ }^{(86)}$.

\section{CONCLUSIONES}

Los estudios demuestran que se ha trabajado con algún detalle sobre las propiedades biológicas de la maca amarilla, la maca negra y la maca roja, y se ha mostrado diferencias entre ellas, pues la maca negra tiene mejores efectos en el conteo de espermatozoides, la memoria y el aprendizaje, el control de la glucosa y la resistencia física, en tanto que la maca roja tiene efectos sobre la hiperplasia benigna de próstata y en la osteoporosis; sin embargo, existen muchas otras variedades que aún requieren ser evaluadas. Es difícil precisar un mecanismo de acción para las propiedades de la maca; los estudios parecen demostrar que el efecto no es a través de cambios hormonales sino más bien posreceptor o, en casos de la osteoporosis y la hiperplasia de próstata, podría estar actuando como modulador selectivo del receptor beta de estrógenos. Esta hipótesis, sin embargo, requiere ser confirmada en futuros estudios.

La demanda interna y externa por la maca sigue en aumento, sin embargo, hay preocupación principalmente en el mercado europeo sobre el contenido de alcaloides de la maca y un efecto negativo en la salud. Por ello, es de suma importancia y de alta relevancia para el país desarrollar estudios donde se puedan evaluar las fracciones con alcaloides y sin alcaloides de la maca que permitan dar a conocer en cuál de ellas se encuentra la actividad biológica de la maca. Es importante, igualmente, desarrollar ensayos clínicos que permitan verificar en humanos las propiedades biológicas de la maca observadas en estudios experimentales.

Agradecimiento: a la Srta. Gissell Alessandra Manrique por su apoyo técnico en la preparación del manuscrito.

Fuente de financiamiento: canon, sobrecanon y regalías mineras de la Universidad Nacional Daniel Alcides Carrión (Cerro de Pasco).

Conflictos de interés: GFG es gerente general de Cayenatur EIRL, empresa dedicada a productos naturales. Los otros autores declaran no tener conflicto de interés para este artículo.

\section{REFERENCIAS BIBLIOGRÁFICAS}

1. Gonzales GF, Gonzales C, Gonzales-Castañeda C. Lepidium meyenii (Maca): a Plant from the Highlands of Peru - from tradition to science. Res Complem Med. 2009; 16(6):373-80. doi: $10.1159 / 000264618$.

2. Cieza de León P: Chronicle of Peru. First Part. London: Hakluyt Society; 1553.

3. Cobo B. History of the New World. Madrid: Biblioteca de Autores Españoles; 1956.

4. Tello J, Hermann M, Calderón A. La maca (Lepidium meyenii Walp.) cultivo alimenticio potencial para las zonas altoandinas. Bol Lima. 1992;14:59-66.

5. Gonzales GF, Miranda S, Nieto J, Fernandez G, Yucra S, Rubio J, et al. Red Maca (Lepidium meyenii) reduced prostate size in rats. Reprod Biol Endocrinol. 2005;3(1):5

6. Zhao J, Avula B, Chan M, Clément C, Kreuzer M, Khan IA. Metabolomic differentiation of maca (Lepidium meyenii) accessions cultivated under different conditions using NMR and chemometric analysis. Planta Med. 2012;78(1):90-101. doi: $10.1055 / \mathrm{s}-0031-1280117$.

7. Gonzales GF. Maca de la Tradición a la Ciencia. Lima: Universidad Peruana Cayetano Heredia; 2006.

8. Melnikovova I, Havlik J, FernandezCusimamani E, Milella L. Macamides and fatty acid content comparison in maca cultivated plant under field conditions and greenhouse. Bol Latinoam Caribe Plant Med Aromat. 2012;11(5):420-7.

9. Valentová K, Buckiova D, Kren V, Peknicova J, Ulrichova J, Simanek V. The in vitro biological activity of Lepidium meyenii extracts. Cell Biol Toxicol. 2006;22(2):91-9.

10. Piacente S, Carbone V, Plaza A, Zampelli A, Pizza C. Investigation of the tuber constituents of maca (Lepidium meyenii walp). J Agric Food Chem. 2002;50(20):5621-5.

11. Castaño MP. Maca (Lepidium peruvianum Chacon): composición química y propiedades farmacológicas. Rev Fitoterapia. 2008;8(1):23-30.
12. McCollom MM, Villinski JR, McPhail KL, Craker LE, Gafner S. Analysis of macamides in samples of maca (Lepidum meyenii) by HPLC-UV-MS/MS. Phytochemical Anal. 2005;16(6):463-9.

13. Zhao J, Muhammad I, Dunbar DCh, Mustafa J, Khan IA. New alkamides from maca (Lepidium meyenii). J Agric Food Chem. 2005;53(3):690-3.

14. Yabar E, Pedreschi R, Chirinos R, Campos D. Glucosinolate content and myrosinase activity evolution in three maca (Lepidium meyenii Walp.) ecotypes during preharvest, harvest and postharvest drying. Food Chem. 2011;127(4):1576-83.

15. Gonzales GF. Maca: Del alimento perdido de los Incas al milagro de los Andes: Estudio de seguridad alimentaria y nutricional. Segurança Alimentar e Nutricional, Campinas. 2010;17(1):16-36.

16. Valerio LG, Gonzales GF. Toxicological aspects of the South American herbs cat's claw (Uncaria tomentosa) and Maca (Lepidium meyenii) : a critical synopsis. Toxicol Rev. 2005;24(1):11-35. 
17. Ahmadi H, Daneshmand S. Androgen deprivation therapy: evidencebased management of side effects. BJU Int. 2013;111(4):543-8.

18. Zheng BL, He K, Kim CH, Rogers L, Yu S, Huang ZY, et al. Effect of a lipidic extract from Lepidium meyenii on sexual behavior in mice and rats. Urology. 2000;55(4):598-602.

19. Cicero AF, Bandieri E, Arletti R. Lepidium meyenii Walp. improves sexual behaviour in male rats independently from its action on spontaneous locomotor activity. J Ethnopharmacol. 2001;75(2-3):225-9.

20. Cicero AF, Piacente S, Plaza A, Sala E, Arletti R, Pizza C. Hexanic Maca extract improves rat sexual performance more effectively than methanolic and chloroformic Maca extracts. Andrologia. 2002;34(3):177-9.

21. Lentz A, Gravitt K, Carson CC, Marson L. Acute and chronic dosing of Lepidium meyenii (Maca) on male rat sexual behavior. J Sex Med. 2007;4(2):332-9.

22. Clément C, Kneubühler J, Urwyler A, Witschi U, Kreuzer M. Effect of maca supplementation on bovine sperm quantity and quality followed over two spermatogenic cycles. Theriogenology. 2010;74(2):173-83.

23. Gonzales GF, Córdova A, Vega K, Chung A, Villena A, Góñez C, et al. Effect of Lepidium meyenii (MACA) on sexual desire and its absent relationship with serum testosterone levels in adult healthy men. Andrologia. 2002;34(6):367-72.

24. Stone M, Ibarra A, Roller M, Zangara A, Stevenson E. A pilot investigation into the effect of maca supplementation on physical activity and sexual desire in sportsmen. J Ethnopharmacol. 2009;126(3):574-6.

25. Zenico T, Cicero AF, Valmorri L, Mercuriali M, Bercovich E. Subjective effects of Lepidium meyenii (Maca) extract on well-being and sexual performances in patients with mild erectile dysfunction: a randomised, double-blind clinical trial. Andrologia. 2009;41(2):95-9.

26. Shin BC, Lee MS, Yang FJ, Lim HS, Ernst E. Maca (L. meyenii) for improving sexual function: a systematic review. BMC Complement Alter Med. 2010;10:44. doi:10.1186/1472-688210-44.
27. Brooks NA, Wilcox G, Walker KZ, Ashton JF, Cox MB, Stojanovska L. Beneficial effects of Lepidium meyenii (Maca) on psychological symptoms and measures of sexual dysfunction in postmenopausal women are not related to estrogen or androgen content. Menopause. 2008;15(6):1157-62.

28. Dording CM, Fisher L, Papakostas G, Farabaugh A, Sonawalla S, Fava M, et al. A double blind, randomized, pilot dose-finding study of maca root $(L$. meyenii) for the management of SSRI induced sexual dysfunction. CNS Neurosci Ther. 2008;14(3):182-91.

29. Gonzales GF, Ruiz A, Gonzales C, Villegas L, Cordova A. Effect of Lepidium meyenii (maca) roots on spermatogenesis of male rats. Asian J Androl. 2001; $3(3): 231-3$.

30. Gonzales GF, Rubio J, Chung A, Gasco M, Villegas L. Effect of alcoholic extract of Lepidium meyenii (Maca) on testicular function in male rats. Asian J Androl. 2003;5(4):349-52.

31. Chung F, Rubio J, Gonzales C, Gasco M, Gonzales GF. Dose-response effects of Lepidium meyenii (Maca) aqueous extract on testicular function and weight of different organs in adult rats. J Ethnopharmacol. 2005;98(1-2):143-7.

32. Gonzales C, Rubio J, Gasco M, Nieto J, Yucra S, Gonzales GF. Effect of short-term and long-term treatments with three ecotypes of Lepidium meyenii (MACA) on spermatogenesis in rats. J Ethnopharmacol. 2006;103(3):44854 .

33. Gasco M, Aguilar J, Gonzales GF. Effect of chronic treatment with three varieties of Lepidium meyenii (Maca) on reproductive parameters and DNA quantification in adult male rats. Andrologia. 2007;39(4):151-8.

34. Yucra S, Gasco M, Rubio J, Nieto J, Gonzales GF. Effect of different fractions from hydroalcoholic extract of Black Maca (Lepidium meyenii) on testicular function in adult male rats. Fertil Steril. 2008;89(5 Suppl):1461-7.

35. Gonzales GF, Gonzales-Castañeda C, Gasco M. A mixture of extracts from Peruvian plants (black maca and yacon) improves sperm count and reduced glycemia in mice with streptozotocininduced diabetes. Toxicol Mech Methods. 2013; 23(7):509-18.

36. Gonzales GF, Córdova A, Gonzales C, Chung A, Vega K, Villena A. Lepidium meyenii (Maca) improved semen parameters in adult men. Asian J Androl. 2001;3(4):301-3 .

37. Gonzales GF, Córdova A, Vega K, Chung A, Villena A. Effect of Lepidium meyenii (maca), a root with aphrodisiac and fertility-enhancing properties, on serum reproductive hormone levels in adult healthy men. J Endocrinol. 2003;176:163-8.

38. Bogani P, Simonini F, Iriti M, Rossoni M, Faoro F, Poletti A, et al. Lepidium meyenii (maca) does not exert direct androgenic activities. J Ethnopharmacol. 2006;104:415-7.

39. Oshima M, Gu Y, Tsukada S. Effects of Lepidium meyenii Walp and Jatropha macrantha on blood levels of estradiol$17 \beta$, progesterone, testosterone and the rate of embryo implantation in mice. J Vet Med Sci. 2003;65(10):1145-6.

40. Ruiz-Luna AC, Salazar S, Aspajo NJ, Rubio J, Gasco M, Gonzales GF. Lepidium meyenii (Maca) increases litter size in normal adult female mice. Reprod Biol Endocrinol. 2005;3(1):16.

41. Gasco M, Yucra S, Rubio J, Gonzales GF. Lepidium meyenii (Maca) varieties did not alter female reproductive parameters in adult male rats. J Complem Integr Med. 2008;5(1).

42. D’Arrigo G, Benavides V, Pino J. Evaluación preliminar del efecto de Lepidium meyenii Walp en el desarrollo embrionario de ratón. Rev Per Biol. 2004;11(1):103-6.

43. Alvarez CJ. Utilización de diferentes niveles de Maca en la fertilidad de cobayos [Tesis de Bachiller]. Pasco, Perú: Facultad de Ciencias Agropecuarias, Universidad Nacional Daniel Alcides Carrión; 1993.

44. Lee KJ, Dabrowski K, Rinchard J, Gomez C, Guz L, Vilchez C. Supplementation of maca (Lepidium meyenii) tuber meal in diets improves growth rate and survival of rainbow trout Oncorbynchus mykiss (Walbaum) alevins and juveniles. Aquaculture Res. 2004;35(3):215-23.

45. Lee KJ, Dabrowski K, Sandoval M, Miller MJS. Activity-guided fractionation of phytochemicals of maca meal, their antioxidant activities and effects on growth, feed utilization, and survival in rainbow trout (Oncorhynchus mykiss) juveniles. Aquaculture. 2005;244:293-301.

46. Thigpen JE, Haseman JK, Saunders HE, Setchell KD, Grant MG, For- 
sythe DB. Dietary phytoestrogens accelerate the time of vaginal opening in immature CD-1 mice. Comp Med. 2003;53(6):607-15.

47. Meissner HO, Mrozikiewicz P, Bobkiewicz-Kozlowska T, Mscisz A, Kedzia B, Lowicka A, et al. HormoneBalancing Effect of Pre-Gelatinized Organic Maca (Lepidium perwianum Chacon): (I) Biochemical and Pharmacodynamic Study on Maca using Clinical Laboratory Model on Ovariectomized Rats. Int J Biomed Sci. 2006;2(3):260-72.

48. Gonzales GF, Nieto J, Rubio J, Gasco M. Effect of Black Maca (Lepidium meyenii) on one spermatogenic cycle in rats. Andrologia. 2006;38(5):166-72.

49. Rubio J, Riqueros MI, Gasco M, Yucra S, Miranda S, Gonzales GF. Lepidium meyenii (Maca) reversed the lead acetate induced -- damage on reproductive function in male rats. Food Chem Toxicol. 2006;44(7):1114-22.

50. Gonzales GF, Gasco M, Córdova A, Chung A, Rubio J, Villegas L. Effect of Lepidium meyenii (Maca) on spermatogenesis in male rats acutely exposed to high altitude $(4340 \mathrm{~m})$. J Endocr. 2004;180(1):87-95.

51. Gonzales GF, Chung FA, Miranda S, Valdez LB, Zaobornyj T, Boveris A. Heart mitochondrial nitric oxide synthase is upregulated in male rats exposed to high altitude $(4,340 \mathrm{~m})$. Am J Physiol 2005;288(6):H2568-73.

52. Meissner HO, Reich-Bilinska H, Mscisz A, Kedzia B. Therapeutic Effects of Pre-Gelatinized Maca (Lepidium Peruvianum Chacon) used as a NonHormonal Alternative to HRT in Perimenopausal Women - Clinical Pilot Study. Int J Biomed Sci. 2006;2(2):14359.

53. Meissner HO, Mscisz A, Reich-Bilinska H, Kapczynski W, Mrozikiewicz P, Bobkiewicz-Kozlowska T, et al. Hormone-Balancing Effect of Pre-Gelatinized Organic Maca (Lepidium peruvianum Chacon): (II) Physiological and Symptomatic Responses of EarlyPostmenopausal Women to Standardized doses of Maca in Double Blind, Randomized, Placebo-Controlled, Multi-Centre Clinical Study. Int J Biomed Sci. 2006;2(4):360-74.

54. Rubio J, Caldas M, Dávila S, Gasco M, Gonzales GF. Effect of three different cultivars of Lepidium meyenii
(Maca) on learning and depression in ovariectomized mice. BMC Complement Altern Med. 2006;6:23.

55. Voronina TA, Belopol'skaya MV, Kheyfets IA, Dugina YL, Sergeeva SA, Epshtein OI. Effect of ultralow doses of antibodies to S-100 protein in animals with impaired cognitive function and disturbed emotional and neurological status under conditions of experimental Alzheimer disease. Bull Exp Biol Med. 2009;148(3):533-5.

56. Rubio J, Dang H, Gong M, Liu X, Chen S-L, Gonzales GF. Aqueous and hydroalcoholic extracts of Black Maca (Lepidium meyenii) improve scopolamine-induced memory impairment in mice. Food Chem Toxicol. 2007;45(10):1882-90.

57. Rubio J, Qiong W, Liu X, Jiang Z, Dang $\mathrm{H}$, Chen SL, et al. Aqueous Extract of Black Maca (Lepidium meyenii) on Memory Impairment Induced by Ovariectomy in Mice. Evid Based Complement Alternat Med. 2011;2011:253958. doi: 10.1093/ ecam/nen063.

58. Rubio J, Yucra S, Gasco M, Gonzales GF. Dose-response effect of black maca (Lepidium meyenii) in mice with memory impairment induced by ethanol. Toxicol Mech Methods. 2011;21(8):628-34.

59. Oré R, Suárez S, Rojas L, Valdivieso R, Oriondo R, Tapia F, et al. Efecto del extracto acuoso de maca sobre la función cognitiva en ratas recién destetadas. An Fac Med. 2011;72(1):13-6.

60. Pino-Figueroa A, Nguyen D, Maher TJ. Neuroprotective effects of Lepidium meyenii (Maca). Ann N Y Acad Sci. 2010;1199:77-85.

61. Suarez S, Oré R, Armas I, Rojas L, Trabucco J. Extracto acuoso de Lepidium meyenii WALP (maca) y su papel como adaptógeno en un modelo animal de resistencia física. An Fac Med. 2009;70(3):181-5.

62. Shin S, Park D, Jeon JH, Joo SS, Kim YB, Kand HG. Gelatinized and fermented powders of Lepidium meyenii (Maca) improve physical stamina and epididymal sperm counts in male mice. J Embryo Transf. 2008;23:283-9.

63. Zheng BL, He K, Hwang ZY, Lu Y, Yan SJ, Kim CH, et al. Effect of aqueous extract from Lepidium meyenii on mouse behavior in forced swimming test. In C.-T. Ho \& Q. Y. Zheng
(Eds.). Quality Management of Nutraceuticals, American Chemical Society Symposium Series 2002; (vol. 803,pp. 258-268). Washington, DC: Oxford University Press.

64. Choi EH, Kang JI, Cho JY, Lee SH, Kim TS, Yeo IH, et al. Supplementation of standardized lipid-soluble extract from maca (Lepidium meyenii) increases swimming endurance capacity in rats. J Funct Foods. 2012;4:568-73.

65. Ronceros G, Ramos W, Garmendia F, Arroyo J, Gutiérrez J. Eficacia de la maca fresca (Lepidium meyenii WALP) en el incremento del rendimiento físico de deportistas en altura. An Fac Med. 2005;66(4)269-73.

66. Nicholson TM, Ricke WA. Androgens and estrogens in benign prostatic hyperplasia: past, present and future. Differentiation. 2011;82(4-5):184-99.

67. Shrivastava A, Gupta VB. Various treatment options for benign prostatic hyperplasia: a current update. J Medlife Health. 2012;3(1):10-9.

68. Gasco M, Villegas L, Villegas L, Rubio J, Gonzales GF. Dose-response effect of Red Maca (Lepidium meyenii) on benign prostatic hyperplasia induced by testosterone enanthate. Phytomedicine. 2007; 14(7-8):460-4.

69. Gonzales C, Leiva-Revilla J, Rubio J, Gasco M, Gonzales GF. Effect of red maca (Lepidium meyenii) on prostate zinc levels in rats with testosteroneinduced prostatic hyperplasia. Andrologia 2012;44 Suppl 1:362-9.

70. Briot K, Cortet B, Thomas T, Audran M, Blain H, Breuil V, et al. 2012 update of French guidelines for the pharmacological treatment of postmenopausal osteoporosis. Joint Bone Spine. 2012;79(3):304-13.

71. Molla MD, Hidalgo-Mora JJ, Soteras MG. Phytotherapy as alternative to hormone replacement therapy. Front Biosci (Schol Ed). 2011;3:191-204.

72. Zhang Y, Yu L, Ao M, Jin W. Effect of etanol extract of Lepidium meyenii Walp. on osteoporosis in ovariectomized rat. J Ethnopharmacol. 2006;105(1-2): 274-9.

73. Gonzales C, Cárdenas-Valencia I, Leiva-Revilla J, Anza-Ramirez C, Rubio J, Gonzales GF. Effects of different varieties of Maca (Lepidium meyenii) on bone structure in ovariectomized rats. Forsch Komplementmed. 2010;17(3):137-43. 
74. Harris HA. Estrogen receptor-beta: recent lessons from in vivo studies. Mol Endocrinol. 2007;21(1):1-13.

75. Rodrigo ME, Valdivieso R, Suárez S. Disminución del daño oxidativo y efecto hipoglicemiante de la maca (Lepidium meyenii Walp) en ratas con diabetes inducida por streptozotocina. An. Fac. Med. 2011;72(1):7-11.

76. Vecera R, Orolin J, Skottová N, Kazdová L, Oliyarnik O, Ulrichová J, et al. The influence of maca (Lepidium meyenii) on antioxidant status, lipid and glucose metabolism in rat. Plant Foods Hum Nutr. 2007;62(2):59-63.

77. Valentová K, Stejskal D, Bartek J, Dvorácková S, Kren V, Ulrichová J, et al. Maca (Lepidium meyenii) and yacon (Smallanthus sonchifolius) in combination with silymarin as food supplements: in vivo safety assessment. Food Chem Toxicol. 2008;46(3):1006-13.

78. Ranilla LG, Kwon YI, Apostolidis E, Shetty K. Phenolic compounds, antioxidant activity and in vitro inhibitory potential against key enzymes relevant for hyperglycemia and hypertension of commonly used medicinal plants, herbs and spices in Latin America. Bioresour Technol. 2010;101(12):4676-89.
79. Gonzales GF, Gasco M, Lozada-Requena I.. Role of maca (Lepidium meyenii) consumption on serum interleukin-6 levels and health status in populations living in the Peruvian Central Andes over $4000 \mathrm{~m}$ of altitude. Plant Foods Hum Nutr. 2013;68(4): 347-51.

80. Oré R, Mayorca JR, Valdivieso R, Ronceros G, Ráez E, Durand J, et al. Efectos adversos de la maca y atorvastatina en hígado de ratas hipercolesterolémicas. Rev Soc Quim Peru, 2004;70(1):917.

81. López-Fando A, Gómez-Serranillos MP, Iglesias I, Lock O, Upamayta UP, Carretero ME. Lepidium peruvianum chacon restores homeostasis impaired by restraint stress. Phytother Res. 2004;18(6):471-4.

82. Castillo P, Lock O. Compuestos con actividad antioxidante en la especie Lepidium meyenii (Walp). Rev Soc Quim Peru. 2005;71(4):227-36.

83. Oré MR. Efectos hipolipémicos y antioxidante de Lepidium meyenii Walp en ratas [Tesis para optar el grado académico de Doctor en Ciencias Biológicas]. Lima, Perú: Universidad Nacional Mayor de San Marcos; 2008.
84. Campos D, Chirinos R, Barreto O, Noratto G, Pedreschi R. Optimized methodology for the simultaneous extraction of glucosinolates, phenolic compounds and antioxidant capacity from maca (Lepidium meyenii). Industrial Crops and Products. 2013;49:747-54.

85. Berłowski A, Zawada K, Wawer I, Paradowska K. Antioxidant Properties of Medicinal Plants from Peru. Food and Nutrition Sciences. 2013;4(8A):71-7.

86. Gonzales GF, Vasquez VB, Gasco M. The transillumination technique as a method for the assessment of spermatogenesis using medicinal plants: the effect of extracts of black maca (Lepidium meyenii) and camu camu (Myrciaria dubia) on stages of the spermatogenic cycle in male rats. Toxicol Mech Methods. 2013;23(8):559-65.

Correspondencia: Gustavo Gonzales.

Dirección: Av. Honorio Delgado 430, Lima 31, Perú.

Teléfono 3190000 anexo 2535

Correo electrónico:gustavo.gonzales@upch.pe 